\title{
INFINITE INFRARED REGULARIZATION AND A STATE SPACE FOR THE HEISENBERG ALGEBRA
}

\author{
ANDREAS U. SCHMIDT
}

\begin{abstract}
We present a method for the construction of a Krein space completion for spaces of test functions, equipped with an indefinite inner product induced by a kernel which is more singular than a distribution of finite order. This generalizes a regularization method for infrared singularities in quantum field theory, introduced by G. Morchio and F. Strocchi, to the case of singularites of infinite order. We give conditions for the possibility of this procedure in terms of local differential operators and the Gelfand-Shilov test function spaces, as well as an abstract sufficient condition. As a model case we construct a maximally positive definite state space for the Heisenberg algebra in the presence of an infinite infrared singularity.
\end{abstract}

\section{INTRODUCTION}

A notable case in which some of the abundant singularities of quantum field theory can be treated rigorously is presented by the method of infrared regularization of Morchio and Strocchi [9, 15]. There, the first-order singularity of the two-point function of the massless scalar field in 1+1-dimensional spacetime manifests itself in the nonpositivity of the Wightman inner product on the one-particle space. In momentum space, this two-point function appears as a singular integral kernel which is regularized in the distributional sense as a Cauchy principal value. Since this regularization involves subtraction of values of the test functions at $p=0$, the Wightman inner product induced by the two-point function is clearly no longer positive definite. It turns out that, if the usual positivity axiom of Wightman theory, see [14], is replaced by a weaker Hilbert space structure condition, the construction of a suitable physical state space is still possible. The one-particle space becomes a Krein space, the natural analogon of a Hilbert space in the case of an indefinite inner product, and it is maximal in the sense that there is no larger Krein space closure of the test function space (we refer to Appendix B where some basic notions of, and results on, indefinite inner product spaces are gathered). Thus, no physical information gets lost and one can identify a positive definite physical Hilbert subspace. In fact, in the case treated in [9, 15] the rank of negativity is one, and thus the Krein space is actually a Pontryagin space. The basic principles of this regularization procedure in the rank one case have already been noted in [5] (The author wishes to thank Daniel Dubin for bringing this reference to his attention).

Date: 20th February 2002.

1991 Mathematics Subject Classification. 46C20, 46F05, 47B50, 81T05.

Key words and phrases. Infrared singularity, Gelfand-Shilov space, Heisenberg algebra, indefinite inner product space, Krein space.

This research was supported by a research grant from the Deutsche Forschungsgemeinschaft DFG The author wishes to thank the University of Durban-Westville for its hospitality and South Africa for the weather. Heartfelt thanks go to Erwin Brüning (Durban), Giovanni Morchio, and Franco Strocchi (both Pisa), Daniel Lenz, and Matthias Schork (both Frankfurt am Main) for many useful discussions. 
In [13], we have cast this procedure in abstract form, yielding a method by which every quasi-positive space, i.e., a space with finite rank of negativity, can be completed to a Pontryagin space. By this, we generalized the infrared regularization method to singularities of the type of finite order distributions. On the other hand it is by now well known that constructive approaches to interacting quantum fields generically involve much more singular objects, see [16, 11], namely ultradistributions and even Fourier Hyperfunctions [3, 4]. Thus, it is natural to look for a further generalization of the procedure for finding a maximal Krein space closure starting from a space of test functions with an indefinite inner product induced by a singular kernel, to the case of non-distributional infnite order singularities, and therefore to the case of an infinite number of negative degrees of freedom. This is what we will present in the following.

We illustrate the regularization method 11 by a neat (yet unphysical) model in which infinite order singularities appear naturally, at least on a heuristic level. Namely, we will consider the Schrödinger representation of the Heisenberg algebra on a test function space over $\mathbb{R}$ in the presence of a singularity concentrated at $p=0$. This model will be informally described in the next section, where also some notions needed subsequently are introduced. Further, we will state the main result, which is that the regularization procedure yields a maximal Krein space and in it a largest possible, positive definite, closed subspace, which can sensibly be considered as the 'physical state space' for the Heisenberg algebra of 'observables'.

Section 3 contains the regularization procedure proper. It shows in particular that there is a certain balance that has to be kept between the singularity of the inner product, measured in terms of infinite order (local) differential operators, and the choice of test function space, which we express in terms of the Gelfand-Shilov scheme of spaces, see [6. Chapter IV]. The method itself is, however, general enough to be applied to a much wider class of singularities than infrared ones, and for a lot of other test function spaces.

In Section 4 we formulate abstractly a sufficient condition under which the regularization is guaranteed to work. The conditions we give are not the most general and abstract ones possible, since they reflect the limitations of the procedure of Section 3 Therefore they present no sharp criterion to decide whether an indefinite space admits the construction of a maximal majorant topology by our construction of a Hilbert majorant. Nevertheless, they capture the essential points that enable our construction, and therefore are at least useful to explain the mechanism behind it. Furthermore, our conditions are simple enough to be effective in many concrete cases. We discuss possible further generalizations at the end of Section 4

Appendix $\AA$ contains a simple, concrete construction of certain neutral elements, i.e., vectors with vanishing inner product, which play an important role in the regularization procedure in Section 3 . Appendix[B compiles some basics about indefinite inner product spaces mainly taken from [2].

\section{The Model}

Recently, a comprehensive abstract classification of representations of the Heisenberg algebra on an indefinite inner product space has been worked out

\footnotetext{
${ }^{1}$ We tend to denote the whole process of defining an indefinite inner product by a generalized function and the construction of a maximal Krein space closure as 'regularization'. The first step, which is the traditional regularization of a singular integral, means going only half way toward a physically conclusive result.
} 
by Mnatsakanova, Morchio, Strocchi, and Vernov in [8]. There, it was pointed out that this issue is somewhat more difficult to handle than in the positive definite case, which is covered by the Stone-von Neumann uniqueness theorem, see [12, Chapter IV]. Especially, domain questions appear and the notion of irreducibility has to be reconsidered. Here, we take a different approach in considering a very concrete example where the Heisenberg representation is from the beginning assumed to be the quantum mechanical Schrödinger representation

$$
\hat{q} \stackrel{\text { def }}{=} x \cdot, \quad \hat{p} \stackrel{\text { def }}{=}-i \frac{\mathrm{d}}{\mathrm{d} x},
$$

on a function space over $\mathbb{R}$.

The following discussion will take place in momentum space, and we will notoriously denote the Fourier transforms of functions with $f, g$, etc., and the variable by $p$. Consider the indefinite inner product

$$
\langle f, g\rangle \stackrel{\text { def }}{=}(f, g)_{L^{2}}-\sum_{k=0}^{\infty} c_{k}^{2} \bar{f}^{(k)}(0) g^{(k)}(0),
$$

with real coefficients $c_{k}$ (this will turn out to pose no essential restriction in our case, see the following). It can be formally interpreted as being induced by a generalized function (a kernel) on $\mathbb{R}^{2}$ in the following way:

$$
\langle f, g\rangle=\left(\delta\left(p-p^{\prime}\right)-J\left(\partial_{p} \partial_{p^{\prime}}\right) \delta(p) \delta\left(p^{\prime}\right), \bar{f}(p) g\left(p^{\prime}\right)\right) .
$$

Here, the infinite order differential operator $J$ is given by its symbol

$$
J(\xi) \stackrel{\text { def }}{=} \sum_{k=0}^{\infty} c_{k}^{2} \xi^{k} .
$$

The singularity in $\langle.,$.$\rangle can be characterized by the following notion, where we$ have already adapted the conventional notation a bit, so as to conform with our application in Section 3 .

Definition and Remark 2.1 ([ㄸ, 159 pp.]). An entire function $J(\xi)$ in the complex variable $\xi$ is called infra-exponential of order $1 /(2 \delta)$ if it fulfills for every $\varepsilon>0$ an estimate

$$
|J(\xi)| \leq C_{\varepsilon} \mathrm{e}^{\varepsilon|\xi|^{1 /(2 \delta)}},
$$

for some $C_{\varepsilon}>0$. In this case, the coefficients $c_{k}^{2}$ of the Taylor series of $J$ satisfy the following upper bounds: For every $D>0$ exists a $\theta \in(0,1)$ and a $C>0$ such that

$$
\left|c_{k}^{2}\right| \leq C \frac{\theta^{k}}{D^{k} \mathrm{e}^{2 k \delta} k^{2 k \delta}} .
$$

Now, our first concern is on which test function space the inner product can be defined. To this end, we use the Gelfand-Shilov scheme for the classification of spaces of smooth functions, see [6. Chapter IV]. For $0 \leq \alpha, \beta \leq \infty$ the space $\mathscr{S}_{\alpha}^{\beta}(\mathbb{R})$ consists of smooth functions $f$ on $\mathbb{R}$ satisfying estimates

$$
\left|p^{q} f^{(k)}(p)\right| \leq C A^{q} B^{k} q^{q \alpha} k^{k \beta} .
$$

We need in fact only consider the regularity of the functions in $\mathscr{S}_{\alpha}^{\beta}$ at the origin, which is expressed in the following basic estimate: There exists a $B>0$ such that for all $\rho>0$ and a constant $C_{f}$ depending on $f$ we have

$$
\left|f^{(k)}(0)\right| \leq C_{f}(B+\rho)^{k} k^{k \beta} .
$$

It is apparent from (3) and (4) that the indefinite inner product is well-defined on $\mathscr{S}_{\alpha}^{\beta}$ by (1) as the distribution (2), whenever $J$ is infra-exponential of order 
$\leq 1 /(2 \beta)$. We denote by $\mathcal{V}=\mathcal{V}_{J}(\alpha, \beta ; \rho)$ a space $\mathscr{S}_{\alpha}^{\beta}$ equipped with an indefinite inner product (1) defined by an infra-exponential symbol $J$ of order $1 /(2 \delta)$ for any $\delta \leq \beta$.

Further constraints on the choice of test function space now come from the intended Schrödinger representation of the Heisenberg algebra. As the Heisenberg generators act in momentum space by multiplication with $p$ and differentiation $i \mathrm{~d} / \mathrm{d} p$, it is clear that they will not be symmetric operators with respect to the indefinite inner product $\langle.,$.$\rangle on the whole space. A represen-$ tation on a subspace of $\mathcal{V}$ acts by symmetric operators only if this subspace consists of functions $f$ such that all derivatives of $f$ vanish at $p=0$. This subspace is at the same time also positive definite. In order that it is also maximal in the sense that there is no larger positive definite subspace in $\mathcal{V}$, one has in fact to assume that the coefficients $c_{k}^{2}$ in equation (1) are strictly positive, i.e., $c_{k} \in \mathbb{R} \backslash\{0\}$ for all $k$, which we do from now on. In turn, this implies that $J\left(\partial_{p} \partial_{p^{\prime}}\right)$ is a properly infinite differential operator and thus the singularity in (2) must be stronger than a finite order distribution. This excludes as test function space any space $\mathscr{S}_{\alpha}^{\infty}$ which allows only distributions of finite order, and thus in particular the Schwartz space $\mathscr{S}=\mathscr{S}_{\infty}^{\infty}$. On the other hand, the very strong singularity of an analytic functional is also excluded: Since for $\beta=1$ the test functions in $\mathcal{V}$ are all analytic in a strip neighbourhood of the real axis, the requirement $f^{(k)}(0)=0$ for all $k$ would lead to the trivial subspace.

After these heuristics, we are ready to state our main result, whose proof will follow in the next section. We will characterize the complete, positive definite representation subspace for the Heisenberg algebra by the Fourier transformation. For that, we need another definition.

Definition 2.2. The space of functions $L_{0}^{2}(\mathbb{R})$ is defined as

$$
L_{0}^{2}(\mathbb{R}) \stackrel{\text { def }}{=}\left\{f \in L^{2}(\mathbb{R}) \mid \mu^{k}(f)=0, \forall k \in \mathbb{N}_{0}\right\},
$$

where the kth moment $\mu^{k}(f)$ of a function $f \in L^{2}(\mathbb{R})$ is given by

$$
\mu^{k}(f) \stackrel{\text { def }}{=} \int_{\mathbb{R}} x^{k} f(x) \mathrm{d} x,
$$

if it exists for a $k \in \mathbb{N}_{0}$.

Theorem 2.3. Let $0 \leq \alpha \leq \infty, 1<\beta<\infty$. Assume $\delta>\beta$, and let $J$ be infraexponential of order $1 /(2 \delta)$ with strictly positive Taylor coefficients. Then, the space $\mathcal{V}=\mathcal{V}_{J}\left(\alpha, \beta ;(2 \delta)^{-1}\right)$ admits a maximal completion to a Krein space $\mathcal{K}$ with countably infinite rank of negativity. The maximal positive definite subspace of $\mathcal{K}$ which is invariant under the action of the Heisenberg algebra in the Schrödinger representation by selfadjoint operators on it, is the Fourier transform $\mathcal{F} L_{0}^{2}(\mathbb{R})$ of $L_{0}^{2}(\mathbb{R})$.

The appearance of $\delta>\beta$ results from technicalities of the infrared regularization process, as will become clear in the following. This leaves room for improvement. It should be stressed that the diagonal form $\delta\left(p-p^{\prime}\right)$ of (2) outside the singularity $p=p^{\prime}=0$ was chosen to allow for a symmetric action of the Heisenberg generators. The regularization procedure itself is nonetheless rather independent of the structure of the kernel outside the singular points. On the other hand, the discussion in the beginning of this section also points to a principal limitation of the regularization method. If the singularity in a certain point $p_{0}$ is that of a proper analytic functional, i.e., $\delta \leq 1$, and the rank 
of indefiniteness is infinite, regularization is impossible since the positive subspace would be trivial in that case.

The somewhat exotic representation of the Heisenberg algebra above does not fit into the classification of [8], see also [10]. Rather it corresponds to the 'counterexample' in the appendix of [8]. As explained there, $L_{0}^{2}(\mathbb{R})$ naturally decomposes into two irreducible subspaces of 'left-' and 'right-movers', i.e., states with support on the negative, respectively positive half-axis in momentum space, by closure of the domains $\mathcal{V}_{ \pm}=\{f \in \mathcal{V} \mid f(p)=0$ for $p \lessgtr 0\}$ in the Krein topology.

\section{INFINITE INFRARED REGULARIZATION}

In this section we present the proper method for the construction of a Krein space from $\mathcal{V}$. The general strategy is close in spirit to the well-known method for closing a Hilbert space with respect to the action of a given positive bilinear form on it, see [7] Appendix A.2]. The construction of a maximal majorant Hilbert topology for $\mathcal{V}$ leading to a Krein space closure of it, relies mainly on two ingredients: First, the existence of neutral elements within $\mathcal{V}$ which separate the negative degrees of freedom from the rest of the space. Second, there is some 'air' left between the decay of the coefficients $c_{k}^{2}$ defining $\langle.,$.$\rangle via (1), and$ the growth of the Taylor coefficients of the functions in $\mathcal{V}$. This is expressed in equation (3), the assumption $\delta>\beta$ of Theorem 2.3, and (4). To make use of that margin, we define the 'damping coefficients' $\gamma_{k}$ by

$$
\gamma_{k} \stackrel{\text { def }}{=} k^{k \delta} \text {. }
$$

The neutral decomposition elements will be constructed in Appendix $\mathrm{A}$ to fulfill the following demands:

Lemma 3.1. Let $0 \leq \alpha \leq \infty$ and $1<\beta<\infty$. Let there be given a sequence of numbers $c_{k}$ satisfying (3), and let $\gamma_{k}$ be as in (5). Then there exists a sequence of functions $\left\{\chi_{k}\right\}_{k \in \mathbb{N}_{0}} \subset \mathscr{S}_{\alpha}^{\beta}$ with the following properties:

i) $\left\|\chi_{k}\right\|_{L^{2}}^{2}=c_{k}^{2} \gamma_{k}^{2}$.

ii) $\chi_{k}^{(i)}(0)=\delta_{i k} \cdot \gamma_{k}$.

iii) $\left(\chi_{k}, \chi_{l}\right)_{L^{2}}=0$, for all $k \neq l$.

iv) $\left\langle\chi_{k}, \chi_{l}\right\rangle=0$, for all $k, l$.

We denote by $\mathcal{N}$ the linear subspace of $\mathscr{S}_{\alpha}^{\beta}$ generated by $\left\{\chi_{k}\right\}_{k \in \mathbb{N}_{0}}$.

The subspace $\mathcal{N}$ is neutral, $\mathcal{N} \subset \mathcal{V}^{0}$. We also observe that $\mathcal{V}$ is non-degenerate, due to the presence of the $L^{2}$-part in the indefinite product (1). This property will prevail in the closure of $\mathcal{V}$ we construct in the following. Now, every $f \in \mathscr{S}_{\alpha}^{\beta}$ has, for every finite $N \geq 0$, a unique decomposition

$$
f=f^{N+}+\sum_{i=0}^{N} f^{i} \chi_{i}, \quad \text { with } f^{i}=\frac{f^{(i)}(0)}{\gamma_{i}},
$$

and $f^{N+} \in \mathscr{S}_{\alpha}^{\beta}$ is such that $f^{(i)}(0)=0$ for $0 \leq i \leq N$. Furthermore, the sum in the decomposition is clearly in $\mathcal{N}$.

Proposition 3.2. The seminorm $p$ given by the limit

$$
p(f)^{2} \stackrel{\text { def }}{=} \lim _{N \rightarrow \infty}\left[\left\langle f^{N+}, f^{N+}\right\rangle+\sum_{i=0}^{N}\left\{\left|\left\langle f, \chi_{i}\right\rangle\right|^{2}+\left|f^{i}\right|^{2}\right\}\right]
$$

exists and defines a majorant topology $\tau$ on $\mathcal{V}$. 
Proof. Taking Lemma B.3 into account, we have to show that (7), if it is well defined, dominates the inner square. Assuming that the limit in question exists, it is easy to show that $p(f)^{2}$ majorizes the inner square $|\langle f, f\rangle|$ of $f$. Namely, using (6) we can express $\langle f, f\rangle$ as

$$
\langle f, f\rangle=\left\langle f^{N+}, f^{N+}\right\rangle+\sum_{i=0}^{N}\left\{f^{i}\left\langle f, \chi_{i}\right\rangle+\overline{f^{i}}\left\langle\chi_{i}, f\right\rangle\right\}
$$

using property iv) of Lemma 3.1 and the fact that $\left\langle f^{N+}, \chi_{i}\right\rangle=\left\langle f, \chi_{i}\right\rangle$ which follows from it. Now, in every term in the sum above we have the elementary estimate for complex numbers $\left|f^{i}\left\langle f, \chi_{i}\right\rangle+\overline{f^{i}}\left\langle\chi_{i}, f\right\rangle\right| \leq\left|\left\langle f, \chi_{i}\right\rangle\right|^{2}+\left|f^{i}\right|^{2}$. If the first term $\left\langle f^{N+}, f^{N+}\right\rangle$ in (7) has a limit at all, then it tends to $\left(f^{+}, f^{+}\right)_{L^{2}}=$ $\left\|f^{+}\right\|_{L^{2}}^{2} \geq 0$ for a certain $f^{+} \in L^{2}$, showing $p(f)^{2} \geq|\langle f, f\rangle|$ in the limit $N \rightarrow \infty$. It remains to show that all the limits involved in (7) exist. In order to show finiteness of the first term it suffices to show that the decomposition (6) of $f$ converges in $L^{2}(\mathbb{R})$ for $N \rightarrow \infty$, since it then tends to $\left\|f^{+}\right\|_{L^{2}}^{2}$ and is thus necessarily finite as we have just seen. For the sum defining the decomposition, we have by i) of Lemma 3.1

$$
\left\|\sum_{N=0}^{\infty} f^{i} \chi_{i}\right\|_{L^{2}}^{2} \leq \sum_{N=0}^{\infty}\left\|f^{i} \chi_{i}\right\|_{L^{2}}^{2}=\sum_{N=0}^{\infty}\left|f^{(i)}(0) c_{i}\right|^{2}<\infty,
$$

taking (3) and (4) into account, showing that claim. By definition (1) of the inner product, the $i$ th term in the sum in (7) becomes

$$
\begin{aligned}
\left|\left\langle f, \chi_{i}\right\rangle\right|^{2}+\left|f^{i}\right|^{2} & =\left|\left(f, \chi_{i}\right)_{L^{2}}-\sum_{k=0}^{\infty} c_{k}^{2} \bar{f}^{(k)}(0) \chi_{i}^{(k)}(0)\right|^{2}+\left|f^{i}\right|^{2} \\
& \leq\left|\left(f, \chi_{i}\right)_{L^{2}}\right|^{2}+c_{i}^{4} \gamma_{i}^{2}\left|f^{(i)}(0)\right|^{2}+\frac{\left|f^{(i)}(0)\right|^{2}}{\gamma_{i}^{2}} .
\end{aligned}
$$

For the first term we find, using the Cauchy-Schwartz estimate, by (3) and (5), and of course the assumption of Theorem 2.3

$$
\left|\left(f, \chi_{i}\right)_{L^{2}}\right|^{2} \leq\|f\|_{L^{2}}^{2}\left|c_{i}^{2} \gamma_{i}^{2}\right| \leq\|f\|_{L^{2}}^{2} \frac{C \theta^{i}}{D^{i} \mathrm{e}^{2 i \delta}} .
$$

Further using (4), the second term is bounded by

$$
c_{i}^{4} \gamma_{i}^{2}\left|f^{(i)}(0)\right|^{2} \leq \frac{C^{2} C_{f}^{2}}{i^{2 i(\delta-\beta)}}\left(\frac{\theta(B+\rho)}{\mathrm{e}^{2 \delta} D}\right)^{2 i} .
$$

Finally the third term satisfies

$$
\frac{\left|f^{(i)}(0)\right|^{2}}{\gamma_{i}^{2}} \leq \frac{C_{f}^{2}}{i^{2 i(\delta-\beta)}}(B+\rho)^{2 i} .
$$

All three terms decay faster than exponentially in $i$, making the overall sum in (7) convergent in the limit $N \rightarrow \infty$.

Notice that although we chose to see this independently by considering $L^{2}$ convergence, the numerical convergence of the $\left\langle f^{N+}, f^{N+}\right\rangle$-part of the decomposition could have been inferred in the same way as the convergence of the other terms in (7). In fact, one could have inverted the decomposition (6) to yield $f^{N+}=f-\sum_{i=0}^{N} f^{i} \chi_{i}$ and then see the convergence of $\left\langle f^{N+}, f^{N+}\right\rangle$ by majorizing it with the same convergent terms as in the previous proof.

It is apparent from the proof of Proposition 3.2 that the decomposition (6) of $f$ converges in the closure $\overline{\mathcal{V}}^{\tau}$ of $\mathcal{V}$ with respect to $\tau$. In fact, it is easy to see 
that the increments $p\left(f^{i} \chi_{i}\right)^{2}$ decay fast enough to turn the partial sums in the decomposition into a Cauchy sequence. This allows us to write for every $f \in \mathcal{V}$,

$$
f=f^{+}+\sum_{i=0}^{\infty} f^{i} \chi_{i}, \quad \text { with } f^{+} \in \overline{\mathcal{V}}^{\tau} .
$$

We further see, using the joint continuity of $\langle.,$.$\rangle , see Definition B.2 that the$ indefinite inner product $\langle.,$.$\rangle has a unique extension to \overline{\mathcal{V}}^{\tau}$, which we will also denote by $\langle.,$.$\rangle . Thus using (8), equation (7) extends to a definition of a qua-$ dratic normed topology on the $\tau$-complete space $\overline{\mathcal{V}}^{\tau}$, i.e., a Hilbert majorant topology on that space:

Corollary 3.3. On the closure $\mathcal{K} \stackrel{\text { def }}{=} \overline{\mathcal{V}}^{\tau}={\overline{\mathscr{S}_{\alpha}^{\beta}}}^{\tau}$ we define the Hilbert scalar product

$$
(f, g) \stackrel{\text { def }}{=}\left\langle f^{+}, g^{+}\right\rangle+\sum_{i=0}^{\infty}\left\{\left\langle f, \chi_{i}\right\rangle\left\langle\chi_{i}, g\right\rangle+\overline{f^{i}} g^{i}\right\}, \quad \forall f, g \in \mathcal{K} .
$$

We denote the Hilbert norm on $\mathcal{K}$ by $\|.\| \stackrel{\text { def }}{=} p()=.(., .)^{1 / 2}$. In particular, we have the identity $\left\langle f^{+}, f^{+}\right\rangle=\left\|f^{+}\right\|_{L^{2}}^{2}$.

We set

$$
\mathcal{P} \stackrel{\text { def }}{=}\left\{f \in \mathcal{V} \mid f^{(k)}(0)=0, \forall k \in \mathbb{N}_{0}\right\} .
$$

Obviously, $\langle.,$.$\rangle is positive definite on \mathcal{P}$ and equals the $L^{2}$-scalar product on that subspace. The decomposition (8) can now be expressed as follows:

Lemma 3.4. Equation (8) defines a mapping

$$
P: \mathcal{V} \longrightarrow \overline{\mathcal{V}}^{\tau} ; \quad f \longmapsto f^{+},
$$

with the following properties:

i) $P$ is continuous in the topology $\tau$.

ii) $P$ has a continuous extension to $\mathcal{K}$.

iii) $P$ maps $\mathcal{K}$ onto $\overline{\mathcal{P}}^{\tau}$.

iv) $P$ is an orthogonal projection onto $\overline{\mathcal{P}}^{\tau}$ with respect to $(.,$.$) .$

v) The decomposition

$$
\mathcal{K}=\overline{\mathcal{P}}^{\tau} \oplus \overline{\mathcal{N}}^{\tau}
$$

is orthogonal with respect to the scalar product $(.,$.$) (denoted by \oplus$ ).

Proof. To show i) we estimate

$$
\begin{aligned}
\left\|f^{+}\right\|^{2} & =\left\|f^{+}\right\|_{L^{2}}^{2}+\sum_{i=0}^{\infty}\left|\left\langle f^{+}, \chi_{i}\right\rangle\right|^{2}=\left\|f^{+}\right\|_{L^{2}}^{2}+\sum_{i=0}^{\infty}\left|\left(f^{+}, \chi_{i}\right)_{L^{2}}\right|^{2} \\
& \leq\left\|f^{+}\right\|_{L^{2}}^{2}\left(1+\sum_{i=0}^{\infty}\left\|\chi_{i}\right\|_{L^{2}}^{2}\right) \leq\left\|f^{+}\right\|_{L^{2}}^{2}\left(1+\sum_{i=0}^{\infty} c_{i}^{2} \gamma_{i}^{2}\right) \\
& \leq C\left\|f^{+}\right\|_{L^{2}}^{2} \leq C\|f\|^{2} .
\end{aligned}
$$

In the last step we used that we have $\|f\|^{2} \geq\left\langle f^{+}, f^{+}\right\rangle=\left\|f^{+}\right\|_{L^{2}}^{2}$ by Corollary 3.3. Assertion ii) follows from i). By i) and ii) it suffices to show that $f \in \mathcal{V}$ entails $f^{+} \in \overline{\mathcal{P}}^{\tau}$ to show iii). For that, by the second to last inequality mentioned previously, it suffices to approximate $f^{+}$in the $L^{2}$-norm with elements of $\overline{\mathcal{P}}^{\tau}$. Such an approximation can be easily constructed, e.g., as $f_{\varepsilon}^{+}=\left(1-\rho_{\varepsilon}\right) f^{+}$ 
for $\varepsilon \rightarrow 0$, with the cut-off functions $\rho_{\varepsilon}$ of Lemma A.1 That $P: \mathcal{K} \rightarrow \overline{\mathcal{P}}^{\tau}$ is surjective is now clear, since $P$ is the identity on $\overline{\mathcal{P}}^{\tau}$. For $f^{+} \in \mathcal{P}$ and $\chi \in \mathcal{N}$, the scalar product reduces to

$$
\left(f^{+}, \chi\right)=\sum_{i=0}^{\infty}\left\langle f^{+}, \chi_{i}\right\rangle\left\langle\chi_{i}, \chi\right\rangle=0
$$

since $\left\langle\chi_{i}, \chi\right\rangle=0$, and because $f^{+}$and $\chi$ have decompositions with vanishing $\left(f^{+}\right)^{i}$ and $\chi^{+}$, respectively. Continuity of $\langle.,$.$\rangle then implies statement iv). As-$ sertion v) follows from iv) and the fact that the sum in (8) converges to an element of $\overline{\mathcal{N}}^{\tau}$.

To construct the metric operator $J$ that connects the indefinite with the Hilbert scalar product on $\mathcal{K}$, we have to decompose this space somewhat further. To that end, we consider the functionals

$$
F_{i}(f) \stackrel{\text { def }}{=}\left\langle\chi_{i}, f\right\rangle, \quad f \in \mathcal{V},
$$

on $\mathcal{V}$. These functionals are nonzero since $\mathcal{V}$ is non-degenerate, they vanish on $\mathcal{N}$, and they are clearly bounded with respect to the norm $p$. In fact, we have $\left|F_{i}(f)\right| / p(f) \leq 1$ for $f \in \mathcal{V}$, by (7). That is, the $F_{i}$ have unique continuations (also denoted by $F_{i}$ ) to $\mathcal{K}$ by the Hahn-Banach theorem, and by continuity these satisfy the same bound $0<\left\|F_{i}\right\| \leq 1$.

Lemma 3.5. The uniquely determined vectors $v_{i} \in \mathcal{K}$ which represent $F_{i}$ via $F_{i}(f)=\left(v_{i}, f\right)$ for all $f \in \mathcal{K}$ are actually contained in $\overline{\mathcal{P}}^{\tau}$.

Proof. That the vectors $v_{i}$ exist and are unique in $\mathcal{K}$ follows from Riesz' representation theorem applied to the bounded linear functionals $F_{i}$ on the Hilbert space $\mathcal{K}$. We have to show that they are in $\overline{\mathcal{P}}^{\tau}$. Choose a sequence $\left\{v_{i n}\right\}_{n \in \mathbb{N}}$ in $\mathcal{V}$ that approximates $v_{i}$, i.e., $\left\|v_{i}-v_{\text {in }}\right\| \rightarrow 0$ for $n \rightarrow \infty$. Using the decomposition (8) for the $v_{i n}$ we calculate (adopting Einstein's summation convention for repeated upper and lower indices)

$$
\begin{aligned}
\left\|v_{i}-v_{i n}\right\|^{2}= & \left(v_{i}-\left(v_{i n}\right)^{+}-\left(v_{i n}\right)^{j} \chi_{j}, v_{i}-\left(v_{i n}\right)^{+}-\left(v_{i n}\right)^{k} \chi_{k}\right) \\
= & \left\|v_{i}-\left(v_{i n}\right)^{+}\right\|^{2} \\
& -\left(v_{i}-\left(v_{i n}\right)^{+}-\left(v_{i n}\right)^{j} \chi_{j},\left(v_{i n}\right)^{k} \chi_{k}\right)-\left(\left(v_{i n}\right)^{j} \chi_{j}, v_{i}-\left(v_{i n}\right)^{+}\right) \\
= & \left\|v_{i}-\left(v_{i n}\right)^{+}\right\|^{2} \\
& -\left(v_{i}-v_{i n},\left(v_{i n}\right)^{j} \chi_{j}\right)-\left(\left(v_{i n}\right)^{j} \chi_{j}, v_{i}\right)+\left(\left(v_{i n}\right)^{j} \chi_{j},\left(v_{i n}\right)^{+}\right) .
\end{aligned}
$$

The last term on the right-hand side vanishes for all $n$ due to Lemma 3.4 v). The third term is zero since $\left(v_{i}, \chi_{j}\right)=F_{i}\left(\chi_{j}\right)=\left\langle\chi_{i}, \chi_{j}\right\rangle=0$. We use the Cauchy-Schwartz estimate for the scalar product $(.,$.$) and the fact that \left\|\chi_{i}\right\|=$ $\left|\left(\chi_{i}\right)^{i}\right|=\left|\chi_{i}^{(i)}(0) / \gamma_{i}\right|=1$ to estimate the second term as follows:

$$
\left|\left(v_{i}-v_{i n},\left(v_{i n}\right)^{j} \chi_{j}\right)\right| \leq\left\|v_{i}-v_{i n}\right\| \sum_{j=0}^{\infty}\left|\left(v_{i n}\right)^{j}\right| \leq C\left\|v_{i}-v_{i n}\right\|,
$$

with some constant $C>0$ independent of $n$. In fact, since $\left(v_{i n}\right)^{j}=v_{i n}^{(j)}(0) / \gamma_{j}$, and using (4) and (5) we see that the sum is finite for all $n$. Since the sequence $v_{i n}$ is convergent in the norm $p$ and by definition (7) of this norm, the sum must actually converge and therefore admits a global bound $C$ as above. In conclusion, since $v_{i n}$ is $\tau$-convergent to $v_{i}$, i.e., $\left\|v_{i}-v_{i n}\right\| \rightarrow 0$ for $n \rightarrow \infty$, we must have $\left\|v_{i}-\left(v_{i n}\right)^{+}\right\| \rightarrow 0$ by necessity, and thus already $P v_{i n}$ is $\tau$-convergent to $v_{i}$. This shows the claim. 
The basic properties of the $v_{i}$ are collected in the next lemma.

Lemma 3.6. The vectors $v_{i}$ have the following properties:

i) $\left\langle\chi_{i}, v_{i}\right\rangle=\left(v_{i}, v_{i}\right)=1$.

ii) $\left(v_{i}, v_{j}\right)=\left\langle\chi_{i}, v_{j}\right\rangle=0$ for $i \neq j$.

iii) $\left\langle v_{i}, v_{i}\right\rangle=0$.

iv) $\left\langle v_{i}, f\right\rangle=f^{i}$ for all $f \in \mathcal{V}$.

Proof. Statement i) is clear from the defining property of $v_{i}$, except for the last equality that says $\left\|v_{i}\right\|=1$. This will soon turn out to be true. Let $\left\{v_{i n}\right\}_{n \in \mathbb{N}} \subset \mathcal{P}$ be a sequence converging to $v_{i}$ in $\mathcal{K}$, which exists by Lemma 3.5. Then with (9), and since $\left(v_{i n}\right)^{j}=0$ for all $j$ we have

$$
\frac{\left\|v_{i n}\right\|^{2}}{\left|\left\langle\chi_{i}, v_{i n}\right\rangle\right|}=\frac{\left\langle v_{i n}, v_{i n}\right\rangle}{\left|\left\langle\chi_{i}, v_{i n}\right\rangle\right|}+1+\frac{\sum_{j \neq i}\left\langle\chi_{j}, v_{i n}\right\rangle}{\left|\left\langle\chi_{i}, v_{i n}\right\rangle\right|} \text {. }
$$

Now $\left|\left\langle\chi_{i}, v_{i n}\right\rangle\right|=\left|\left(v_{i}, v_{i n}\right)\right| \longrightarrow\left\|v_{i}\right\|^{2}$ by i), so that the left-hand side tends to 1 for $n \rightarrow \infty$ (here we assume that the denominators are nonzero which can be achieved by choosing $v_{i n}$ suitably). Since the denominators stay bounded, we must necessarily have $\left|\left\langle\chi_{j}, v_{i n}\right\rangle\right| \longrightarrow 0$ for $j \neq i$ showing ii), and also $\left\langle v_{i n}, v_{i n}\right\rangle \longrightarrow 0$ showing iii) since $v_{i n}$ converges to $v_{i}$ with respect to $\|\|=.p($.) and $p$ majorizes the inner square. Incidentally, this also shows

$$
\sup _{n} \frac{\left|F_{i}\left(v_{i n}\right)\right|}{\left\|v_{i n}\right\|}=\sup _{n} \frac{\left|\left\langle\chi_{i}, v_{i n}\right\rangle\right|}{\left\|v_{i n}\right\|}=1
$$

and this proves the last equality in i), since the norm of $v_{i}$ and that of the linear functional $F_{i}$ coincide by Riesz' theorem. To show iv), we consider again the decomposition (8) of a vector $f \in \mathcal{V}$ which yields

$$
\left\langle v_{i n}, f\right\rangle=\left\langle v_{i n}, f^{+}\right\rangle+f^{i}\left\langle v_{i n}, \chi_{i}\right\rangle+\sum_{j \neq i} f^{j}\left\langle v_{i n}, \chi_{j}\right\rangle .
$$

In this expression we find $\left\langle v_{i n}, f^{+}\right\rangle \longrightarrow 0$, since by iii) $v_{\text {in }}$ converges strongly to 0 in $\overline{\mathcal{P}}^{\tau}$, and due to Lemma 3.4. By arguments similar to that in the proof of Lemma 3.5 the sum stays bounded independently of $n$, and since every single term in it converges to 0 by ii), the sum also tends to 0 . This leaves us with the second term which converges to $f^{i}\left\langle v_{i}, \chi_{i}\right\rangle=f^{i}$ by i). This shows iv).

The $v_{i}$ could be constructed concretely as limits of functions which vanish strongly in the $L^{2}$-sense, as in [9]. Lemma 3.5] and 3.6] allow us to avoid such an explicit construction. The vectors $v_{i}$ are an orthonormal basis of a closed Hilbert subspace of $\mathcal{K}$. This space is isomorphic to the dual space of $\overline{\mathcal{N}}^{\tau}$ by definition of the functionals $F_{i}$ and Lemma 3.5, and we mnemonically denote it by the symbol $\overline{\mathcal{N}}^{\tau\langle *\rangle}$.

Lemma 3.7. Denote by $\mathcal{H}$ the closure $\overline{\mathcal{P}}^{\tau_{+}}$of $\mathcal{P}$ with respect to the topology $\tau_{+}$ induced by the quadratic Hilbert norm $p_{+}(.)^{2} \stackrel{\text { def }}{=}\langle.,$.$\rangle on \mathcal{P}$. The space $\mathcal{K}$ admits the decomposition

$$
\mathcal{K}=\mathcal{H} \oplus \overline{\mathcal{N}}^{\tau\langle *\rangle} \oplus \overline{\mathcal{N}}^{\tau},
$$

orthogonal with respect to $(.$, .).

Proof. First, we must show that the decomposition is indeed possible because $\mathcal{H} \subset \overline{\mathcal{V}}^{\tau}$. To this end, note that the topology $\tau_{+}$is stronger than the restriction of $\tau$ to $\mathcal{P}$. In fact, if a sequence in $\mathcal{P}$ converges in the norm $p_{+}$then it converges in the $L^{2}$-norm by (1), and by the action of the indefinite product on $\mathcal{P}$ it is easy to see that this suffices to ensure convergence in the norm $p$. Now, 
taking Lemma 3.4 v) into account, we have to show that the (.,.)-orthogonal decomposition $\overline{\mathcal{P}}^{\tau}=\mathcal{H} \oplus \overline{\mathcal{N}}^{\tau\langle *\rangle}$ holds. First, observe that the vectors $v_{i}$ form a $\tau$-complete orthonormal system in $\overline{\mathcal{N}}^{\tau\langle *\rangle}$. Now, for $f^{+}, g^{+} \in \mathcal{P}$ we have

$$
\left(f^{+}, g^{+}\right)=\left\langle f^{+}, g^{+}\right\rangle+\sum_{i=0}^{\infty}\left(f^{+}, v_{i}\right)\left(v_{i}, g^{+}\right),
$$

by the definition of $v_{i}$ and (9). This shows that a sequence $\left\{f_{n}^{+}\right\}_{n \in \mathbb{N}}$ in $\mathcal{P}$ converges to a limit $f \in \overline{\mathcal{P}}^{\tau}$ if and only if $p_{+}\left(f_{n}^{+}-f\right) \longrightarrow 0$ and independently the (.,.)-orthogonal projection of $f_{n}^{+}-f$ onto the closed subspace $\overline{\mathcal{N}}^{\tau\langle *\rangle}$ of $\overline{\mathcal{P}}^{\tau}$ tends to zero. Denote by $\overline{\mathcal{N}}^{\tau\langle\perp\rangle}$ the orthogonal complement of $\overline{\mathcal{N}}^{\tau\langle *\rangle}$ in $\mathcal{K}$ with respect to (., .). By the above-given argument, the subset $\mathcal{P} \cap \overline{\mathcal{N}}^{\tau\langle\perp\rangle}$ of $\overline{\mathcal{P}}^{\tau}$ is dense in $\mathcal{H}$ with respect to the topology $\tau_{+}$. This shows that the proposed decomposition is indeed (., .)-orthogonal. In conclusion, a $\tau$-Cauchy sequence in $\mathcal{P}$ can be identified with a pair $\left(f,\left\{\lambda_{i}\right\}_{i \in \mathbb{N}_{0}}\right)$ with an $f \in \mathcal{H}$ and $\lambda_{i}=\left(v, v_{i}\right)$ for some $v \in \overline{\mathcal{N}}^{\tau\langle *\rangle}$. This shows $\overline{\mathcal{P}}^{\tau}=\mathcal{H} \oplus \overline{\mathcal{N}}^{\tau\langle *\rangle}$.

It should be noted that by Lemma 3.6 ii), the vectors $v_{i}$ indeed converge to zero in the topology $\tau_{+}$of $\mathcal{H}$ but are clearly nonzero in $\overline{\mathcal{P}}^{\tau} \subset \mathcal{K}$. Furthermore, $\tau_{+}$ is stronger than the $L^{2}$-topology although $p_{+}\left(f^{+}\right)=\left\|f^{+}\right\|_{L^{2}}$ for $f^{+} \in \mathcal{P}$. We will characterize $\mathcal{H}$ as a function space in the following. We have compiled all information needed to exhibit the Krein space structure of $\mathcal{K}$.

Theorem 3.8. The space $\mathcal{K}$ is a Krein space with countably infinite rank of indefiniteness. Its Hilbert space structure is maximal and given by the metric operator $J: \mathcal{K} \rightarrow \mathcal{K}$, satisfying $\langle.,\rangle=.(., J$.$) . It holds$

$$
J v_{i}=\chi_{i}, J \chi_{i}=v_{i} \text {, and }\left.J\right|_{\mathcal{H}}=\mathbb{I}_{\mathcal{H}},
$$

in the decomposition of Lemma 3.7

Proof. The strategy of the proof will be as follows: The metric operator exists by Proposition B.5. and we have seen in Lemma 3.7 that we can write down its action in the decomposition $\mathcal{K}=\mathcal{H} \oplus \overline{\mathcal{N}}^{\tau\langle *\rangle} \oplus \overline{\mathcal{N}}^{\tau}$. We can then explicitly demonstrate that the operator $J$ on $\mathcal{K}$ acts as stated. This special form of $J$ immediately implies that it is a bounded, completely invertible operator on $\mathcal{K}$. Thus by Proposition B.7 $\mathcal{K}$ is a Krein space and since $J^{-1}=J$ is also bounded, its Hilbert space structure $(\mathcal{K}, J)$ is maximal by LemmaB.8 Now, by definition of the $v_{i}$ we have $\left\langle f, \chi_{i}\right\rangle=\left(f, J \chi_{i}\right)=\left(f, v_{i}\right)$ for all $f \in \mathcal{K}$, showing $J \chi_{i}=v_{i}$. On the other hand, by Lemma [3.6 v) and (9) we have $\left\langle f, v_{i}\right\rangle=$ $\left(f, J v_{i}\right)=\overline{f^{i}}=\left(f, \chi_{i}\right)$, showing $J v_{i}=\chi_{i}$. It remains to consider the restriction of $J$ to $\mathcal{H}$. Take $f^{\perp}, g^{\perp} \in \mathcal{P} \cap \overline{\mathcal{N}}^{\tau\langle\perp\rangle}$ (see the proof of Lemma 3.7) and note that $\left(f^{\perp}, g^{\perp}\right)=\left\langle f^{\perp}, g^{\perp}\right\rangle$ for those vectors. Since these vectors are dense in $\mathcal{H}$, it follows that the restriction of $J$ to $\mathcal{H}$ is the identity. This shows the claim.

To conclude the proof of Theorem 2.3. it finally remains to show that $\mathcal{H}$ is the Fourier transform of the space $L_{0}^{2}$ defined in Definition 2.2 Now $\mathcal{F}$ is a topological isomorphism from $\mathscr{S}_{\beta}^{\alpha}$ onto $\mathcal{V}=\mathscr{S}_{\alpha}^{\beta}$ and for $f \in \mathscr{S}_{\beta}^{\alpha}$ we have

$$
i^{k} \widehat{f}^{(k)}(0)=\left.\left(i^{k} \frac{\mathrm{d}^{k}}{\mathrm{~d} \xi^{k}} \int_{\mathbb{R}} \mathrm{e}^{-i x \xi} f(x) \mathrm{d} x\right)\right|_{\xi=0}=i^{k} \int_{\mathbb{R}}(-i x)^{k} f(x) \mathrm{d} x=\mu^{k}(f) .
$$

By that, the image of $\mathcal{P}$ under $\mathcal{F}^{-1}$ is the subspace of $\mathscr{S}_{\beta}^{\alpha}$ of functions $f$ with $\mu^{k}(f)=0$ for all $k$. Since $\mathscr{S}_{\beta}^{\alpha}$ is dense in $L^{2}$ and the Fourier transformation is an $L^{2}$-isometry, we can see $\mathcal{H}=\mathcal{F} L_{0}^{2}$. Thus Theorem 2.3 is finally proven. 
If we test the vectors $v_{i}$ with states in the 'physical' subspace $\mathcal{H}$, i.e., the representation space for the Heisenberg-observables, they appear as completely delocalized states. In fact the action of the momentum operator on them is given by Lemma 3.6 v):

$$
\left\langle p v_{i}, f\right\rangle=\left\langle v_{i}, p f\right\rangle=(p f)^{i}=0, \quad \text { for all } f \in \mathcal{H}, i \in \mathbb{N}_{0},
$$

where we denoted the unique extension of the multiplication operator $p$ from $\mathcal{V}$ to $\mathcal{K}$ also by $p$. This is different from the case of one single negative degree of freedom in [9], where the single vector $v_{0}$ turns out to be completely delocalized on the whole Krein state space.

\section{A Condition Sufficient For REgularization}

In this last section, we want to give a set of conditions on a general indefinite inner product space $\mathcal{V}$, that will be sufficient for the regularization procedure to work. We did not put this generalization in the beginning, and then deduced the special case $\mathcal{V}=\mathscr{S}_{\alpha}^{\beta}$ considered previously from it, for two reasons: First and foremost, we wanted to emphasize the case of indefinite inner products generated by singular kernels acting on a test function space, which we think is particularly interesting in view of possible applications in physics. Second, most of the assertions and proofs in Section 3 are already cast abstract enough to be re-used in the proof of the generalized regularization Theorem 4.1] without any modification. Thus, we can stress the essential points that need modification and thereby highlight the principles which put the regularization procedure to work.

Two elements are essential: First, the existence of neutral decomposition elements $\chi_{i}$ that enable us to isolate the positive part of the indefinite product. Second, a certain balance between the growth, respectively, and decay of a) the inner products of vectors in the space with the neutral elements, and b) the coefficients of the linear decomposition of a vector with respect to these. These growth conditions constitute the main difference between the case of finite rank of indefiniteness considered in [13] and the infinite case, where they serve to render the Hilbert majorant topology well-defined in the first place.

Let us now formulate our set of conditions. We assume $\mathcal{V}$ to be a complex linear space with an indefinite inner product $\langle.,$.$\rangle , which shall be non-degenerate.$ Assume that:

0) There exists an orthogonal system $\left\{\widetilde{\chi}_{i}\right\}_{i \in \mathbb{N}_{0}}$ of mutually linearly independent, neutral vectors in $\mathcal{V}$.

1) For all $v \in \mathcal{V}$, the unique decomposition for $N \in \mathbb{N}_{0}$,

$$
v=\widetilde{v}^{N+}+\sum_{i=0}^{N} \widetilde{v}^{i} \widetilde{\chi}_{i}, \quad \widetilde{v}^{i} \in \mathbb{C},
$$

becomes asymptotically positive in the sense that

$$
0 \leq \lim _{N \rightarrow \infty}\left\langle\widetilde{v}^{N+}, \widetilde{v}^{N+}\right\rangle .
$$

2) There exists a sequence of complex numbers $\left\{\gamma_{i}\right\}_{i \in \mathbb{N}_{0}}$ such that both sequences $\left\{\gamma_{i}\left\langle\widetilde{\chi}_{i}, v\right\rangle\right\}$ and $\left\{\widetilde{v}^{i} / \gamma_{i}\right\}$ are in $l^{2}\left(\mathbb{N}_{0}\right)$.

These conditions enable us to prove an equivalent of Proposition 3.2 In fact, setting $\chi_{i} \stackrel{\text { def }}{=} \gamma_{i} \widetilde{\chi}_{i}$, we obtain the anologue of the finite decomposition (6) for a vector $v \in \mathcal{V}$ with coefficients $v^{i}=\widetilde{v}^{i} / \gamma_{i}$. We then have to see that the sum (7) with $f$ replaced by $v$, defining the majorant norm $p(v)^{2}$, converges. The convergence of the asymptotically positive part $\lim _{N \rightarrow \infty}\left\langle v^{N+}, v^{N+}\right\rangle$ then 
follows, as we have already noted after the proof of Proposition 3.2 on page 6 Now, the $i$ th summand in the definition of $p(v)^{2}$ becomes

$$
\left|\left\langle v, \chi_{i}\right\rangle\right|^{2}+\left|v^{i}\right|^{2}=\left|\gamma_{i}\left\langle v, \tilde{\chi}_{i}\right\rangle\right|^{2}+\left|\widetilde{v}^{i} / \gamma_{i}\right|^{2},
$$

and the sum converges due to condition 2). Thus, we get a majorant Hilbert topology $\tau$ on $\mathcal{V}$. A close inspection of the proofs of the various lemmata in Section 3 shows that the only other point which has to be reconsidered is the proof of statement i) of Lemma 3.4 that the mapping $P: v \mapsto v^{+}$is $\tau$-continuous on $\mathcal{K} \stackrel{\text { def }}{=} \overline{\mathcal{V}}^{\tau}$. There, we have utilized the $L^{2}$-norm, but we will see that this can also be shown independently. In fact, we have

$$
\begin{aligned}
\left\|v^{+}\right\|^{2} & =\left(v-\sum_{i=0}^{\infty} v^{i} \chi_{i}, v-\sum_{j=0}^{\infty} v^{j} \chi_{j}\right) \\
& =\|v\|^{2}-2 \operatorname{Re} \sum_{i=0}^{\infty} v^{i}\left(\chi_{i}, v\right)+\sum_{i, j=0}^{\infty} v^{i} \overline{v^{j}}\left(\chi_{i}, \chi_{j}\right) .
\end{aligned}
$$

We use the two consequences $\left(\chi_{i}, \chi_{j}\right)=\delta_{i j}$ and $\left(\chi_{i}, v\right)=\overline{v^{i}}$ of equation (9) in the third and second term respectively to obtain

$$
=\|v\|^{2}-\sum_{i=0}^{\infty}\left|v^{i}\right|^{2} \leq\|v\|^{2},
$$

by definition (7) of $\|v\|^{2}$. Here again, condition 2) ensures the convergence of the sums appearing. From this point, one can proceed word for word as in Section 3 with the definition of the vectors $v_{i}$ and the demonstration of their properties. We finally obtain a generalization of Theorem 3.8 .

Theorem 4.1. Let $\mathcal{V}$ satisfy 0$)-2$ ). Then $\mathcal{K}$ is a Krein space with rank of indefiniteness equal to $\#\left\{\chi_{i} \neq 0\right\}$. Its Hilbert space structure is maximal, and the metric operator $J$ acts as in Theorem 3.8

Note that 0)-2) and Theorem 4.1 are formulated as to cover the cases of finite as well as of infinite rank of indefiniteness. Namely, in case the rank of indefiniteness is $N<\infty$, one can find at most $N$ neutral, linearly independent vectors, and one has to use them all to obtain a decomposition that, as demanded by 1 ), becomes positive (in this case not asymptotically). One then chooses $\widetilde{\chi}_{i}=0$ for $i>N-1$.

We conclude this paper with some comments on the generalized regularization procedure just described. First, the conditions 0)-2) certainly do not present the utmost general ones possible. In particular, one can perhaps replace the neutral orthogonal system of 0 ) by a general system of linear independent vectors which lead to an aymptotically positive decomposition. See [13, Remark A.13], where we describe how to find a maximal neutral orthogonal system in the case of finite rank of negativity. Furthermore, whether $\left\langle v^{+}, v^{+}\right\rangle$ is positive or negative definite is irrelevant, since one can always go over to $-\langle.,$.$\rangle (the so called anti-space of \mathcal{V}$ ). On the other hand, one cannot easily dispense with either of the $l^{2}$-conditions in 2), since they represent rather sharply the convergence conditions that enabled us to construct a majorant. Since we made no presuppositions with respect to $\mathcal{V}$ regarding structure and topology, condition 1) is also indispensible.

In our case of main interest in Section 3 the essence of conditions 0) and 1) are captured in Lemma 3.1 which is proven in the appendix following this section. A similar construction of neutral decomposition elements will also have to be carried out in any other concrete case, and is thus at the very center of 
the regularization procedure, in putting flesh to the bones of the abstract conditions 0) and 1). The construction in Appendix $\mathrm{A}$ may serve as a blueprint for that at least in the case of test function spaces over $\mathbb{R}^{n}$ and inner products generated by kernels whose singularities are 'localized' enough, e.g., concentrated on a compact set. This may justify that we did not delve into a further abstraction of conditions 0 ) and 1 ).

Let us consider an instructive special case. Assume the sequence of coefficients $\left\{\widetilde{v}^{i}\right\}$ is bounded for all $v \in \mathcal{V}$. If there holds an estimate

$$
\left|\left\langle\widetilde{\chi}_{i}, v\right\rangle\right| \leq C(v) i^{-(1+\delta)},
$$

with a constant depending on $v$ and for some $\delta>0$, we can choose

$$
\gamma_{i}=i^{-(1 / 2+\varepsilon)}
$$

for any $0<\varepsilon<\delta$. Such polynomial growth and decay conditions are obviously much weaker than the conditions that were present in the case $\mathcal{V}=\mathscr{S}_{\alpha}^{\beta}$, see, e.g., our choice of $\gamma$ 's in (5). Thus the range of cases covered by Theorem 4.1] is considerably widened in comparison to Theorem 3.8 and Theorem 2.3

The question arises naturally, whether we can find uniform properties on $\mathcal{V}$, as opposed to the pointwise ones 1 ) and 2), that enable regularization. In essence one would look for a simple quantitative measure that tells us when the construction of the majorant is possible. But this is not straightforward. To simplify the discussion, consider the case where $\left\{\widetilde{v}^{i}\right\}$ is bounded in $\mathbb{C}$ for all $v$ (these sets can of course not be uniformly bounded). A simple uniform measure that could replace condition 2) can be formulated in terms of the quantities

$$
\widetilde{\beta}_{i} \stackrel{\text { def }}{=} \sup _{v \in \mathcal{V}, \widetilde{v}^{i}=1}\left|\left\langle\widetilde{\chi}_{i}, v\right\rangle\right| \text {. }
$$

Notice that at least $\tilde{\chi}_{i}$ is in the set over which the supremum is taken, and if this is the only element we have $\widetilde{\beta}_{i}=0$ due to neutrality of that vector. One can then replace 2) by the condition that there shall exist a sequence $\left\{\gamma_{i}^{-1}\right\}$ in $l^{2}\left(\mathbb{N}_{0}\right)$ such that also $\left\{\widetilde{\beta}_{i} \gamma_{i}\right\}$ is in $l^{2}\left(\mathbb{N}_{0}\right)$. This uniform growth condition on $\widetilde{\beta}_{i}$ is however by far too restrictive, since it does not even cover the case considered in Theorem 2.3 The basic reason for this is that in most cases the neutral orthogonal system $\left\{\widetilde{\chi}_{i}\right\}$ does not exhaust the space $\mathcal{V}$ in the sense that a complete orthogonal system exhausts a Hilbert space. The inner products with these vectors do not contain enough information about the whole space, and especially its positive part, to decide sharply whether $\mathcal{V}$ is regularizable. The problem of finding a good abstract definition of what we would like to call 'regularizable inner product spaces' remains therefore open.

\section{Appendix A. Construction of Neutral Decomposition Elements}

In this Appendix, we present a simple construction for the neutral decomposing functions of Lemma 3.1. We point out that different and more refined constructions are surely possible, but the one given in the following suffices for our purpose.

We have to show i)-iii) since iv) follows from them. We prove Lemma 3.1 for $\gamma_{k}=1, \forall k$. The general case follows by multiplication of the functions $\chi_{k}$ constructed subsequently with the given sequence $\gamma_{k}$. The first thing we need to show is that there are enough functions of compact support in $\mathscr{S}_{\alpha}^{\beta}$. For that, we have to consider the spaces $\mathscr{S}_{\alpha}^{\beta, B}$, which constitute $\mathscr{S}_{\alpha}^{\beta}$ as an inductive limit for $B \rightarrow \infty$, see [6, Chapter IV, §3] for their definition. 
Lemma A.1. Let $0 \leq \alpha \leq \infty$ and $1<\beta<\infty$. For $\varepsilon>0$ there exists $B_{\varepsilon}>0$ and a function $\rho_{\varepsilon} \in \mathscr{S}_{\alpha}^{\beta, B_{\varepsilon}}$ such that

$$
\rho_{\varepsilon}(x)= \begin{cases}1, & \text { if }|x|<\varepsilon / 2 \\ 0, & \text { if }|x|>3 \varepsilon / 2 \\ 0 \leq \rho_{\varepsilon}(x) \leq 1, & \text { otherwise }\end{cases}
$$

Proof. Under the given conditions, the function $\rho_{\varepsilon}$ can be constructed using the well-known facts about the Gelfand-Shilov spaces, for which we refer to [6] Chapter IV]. The space $\mathscr{S}_{\alpha}^{\beta}$ contains the space $\mathscr{S}_{0}^{\beta}$ which consists of functions of compact support and is nontrivial for $\beta>1$. Furthermore, for $\phi \in \mathscr{S}_{0}^{\beta}$ we have $\phi^{2} \in \mathscr{S}_{0}^{\beta}$. Thus there exists a $B>0$ and a nonzero function $\phi$ with $\phi(x) \geq 0$ in $\mathscr{S}_{0}^{\beta, B}$, such that $\operatorname{supp} \phi \subset[-R, R]$ for some $R>0$. Then

$$
\phi_{\varepsilon}(x) \stackrel{\text { def }}{=} \frac{\varepsilon}{2 R\|\phi\|_{L^{1}}} \cdot \phi(2 R x / \varepsilon)
$$

is an element of $\mathscr{S}_{0}^{\beta, B_{\varepsilon}}$ for $B_{\varepsilon}=2 R B / \varepsilon$, see [6 p. 158]. It has $L^{1}$-norm 1 and support in $[-\varepsilon / 2, \varepsilon / 2]$. Since convolution with $L^{1}$-functions does not change the regularity, the function $\phi_{\varepsilon} * \chi_{[-\varepsilon, \varepsilon]}$ is an element of $\mathscr{S}_{0}^{\beta, B_{\varepsilon}}$ and therefore $a$ fortiori of $\mathscr{S}_{\alpha}^{\beta, B_{\varepsilon}}$ with all the desired properties.

We set

$$
\kappa_{n}(x) \stackrel{\text { def }}{=} \rho_{1 / 3}(x-n) /\left\|\rho_{1 / 3}\right\|_{L^{2}}, \quad n \in \mathbb{Z} .
$$

Since $\kappa_{n}$ has support in $[n-1 / 2, n+1 / 2]$, we have $\left(\kappa_{i}, \kappa_{j}\right)=0$ for all $i \neq j$. Define a sequence of positive real numbers by

$$
\varepsilon_{i} \stackrel{\text { def }}{=} \frac{1}{3 \mathrm{e}} \prod_{k=0}^{i} \min \left(1, c_{k}^{2}\right)
$$

Set

$$
\delta_{i} \stackrel{\text { def }}{=} \frac{x^{i}}{i !} \rho_{\varepsilon_{i}}
$$

Furthermore, for $i \neq j$ define

$$
k_{i j} \stackrel{\text { def }}{=} \operatorname{sign}(i-j) \sqrt{\left(\delta_{j}, \delta_{i}\right)_{L^{2}}} .
$$

We use the following enumeration for the off-diagonal entries of an infinite matrix (rows and columns counted from 0 ):

$$
\mathbb{N}_{0}^{2} \backslash \operatorname{diag} \ni(i, j) \longmapsto n(i, j) \stackrel{\text { def }}{=} \begin{cases}\frac{j(j-1)}{2}+i+1, & \text { if } i<j ; \\ n(j, i), & \text { otherwise. }\end{cases}
$$

We use the functions $\delta_{i}$ as building blocks for the desired functions, since they have just the right behaviour at 0 to ensure property ii) of Lemma 3.1 To correct their nonvanishing $L^{2}$-overlap with each other we use the corrective

$$
K_{i} \stackrel{\text { def }}{=} \sum_{j \neq i} k_{i j} \kappa_{n(i, j)} .
$$

We must show that this is possible, i.e., that $\left\|\delta_{i}+K_{i}\right\|_{L^{2}}^{2}$ does not exceed $c_{i}^{2}$, in order to satisfy i). We have

$$
\left\|\delta_{i}+K_{i}\right\|_{L^{2}}^{2}=\left\|\delta_{i}\right\|_{L^{2}}^{2}+\left\|K_{i}\right\|_{L^{2}}^{2}=\sum_{j=0}^{\infty}\left|\left(\delta_{i}, \delta_{j}\right)_{L^{2}}\right| .
$$


The terms in the sum allow for the basic (yet very coarse) estimate

$$
\left|\left(\delta_{i}, \delta_{j}\right)_{L^{2}}\right| \leq \frac{2}{i ! j !}\left(\frac{3}{2} \min \left(\varepsilon_{i}, \varepsilon_{j}\right)\right)^{i+j+1}
$$

by construction of $\delta_{i}$. Using (耳) we have $\min \left(\varepsilon_{i}, \varepsilon_{j}\right) \leq \frac{1}{3 \mathrm{e}} \min \left(c_{i}^{2}, 1\right)$ and therefore we can estimate under the additional assumption $c_{i}^{2} \leq 1$ :

$$
\begin{aligned}
\left\|\delta_{i}+K_{i}\right\|_{L^{2}}^{2} & \leq \sum_{j=0}^{\infty} \frac{2}{i ! j !}\left(\frac{c_{i}^{2}}{2 \mathrm{e}}\right)^{i+j+1} \leq \frac{2}{i !}\left(\frac{c_{i}^{2}}{2 \mathrm{e}}\right)^{i+1} \sum_{j=0}^{\infty} \frac{c_{i}^{2 j}}{j !} \\
& =\frac{2}{i !}\left(\frac{c_{i}^{2}}{2 \mathrm{e}}\right)^{i+1} \mathrm{e}^{c_{i}^{2}} \leq \frac{c_{i}^{2}}{i !(2 \mathrm{e})^{i}} \leq c_{i}^{2} .
\end{aligned}
$$

Now using the function

$$
\nu_{i}=\sqrt{c_{i}^{2}-\left\|\delta_{i}+K_{i}\right\|_{L^{2}}^{2}} \cdot \kappa_{-i}
$$

we can set

$$
\chi_{i} \stackrel{\text { def }}{=} \delta_{i}+K_{i}+\nu_{i} .
$$

We are done if we show that $\chi_{i}$ is well defined as a function in $\mathscr{S}_{\alpha}^{\beta}$, i.e., that the sum $K_{i}$ converges in the topology of the namely space. To that end, we have to show convergence in one of the spaces $\mathscr{S}_{\alpha, A}^{\beta, B}$ which constitute the inductive limit $\mathscr{S}_{\alpha}^{\beta}=\lim _{A, B \rightarrow \infty} \mathscr{S}_{\alpha, A}^{\beta, B}$. We show that the increments in the sum $K_{i}$, namely $k_{i j} \kappa_{n(i, j)}$, decay fast enough in $j$ to turn the sum into a Cauchy sequence in that topology. In the countably normed space $\mathscr{S}_{\alpha, A}^{\beta, B}$, we therefore have to estimate the increments due to the terms in the sum defining $K_{i}$ :

$$
\left\|k_{i j} \kappa_{n(i, j)}\right\|_{\rho, \delta}=\sup _{x, k, q} \frac{\left|x^{k} k_{i j} \kappa_{n(i, j)}\right|}{(A+\rho)^{k}(B+\delta)^{q} k^{k \alpha} q^{q \beta}}, \quad \text { with } \rho, \delta>0 .
$$

We first argue that this quantity can be estimated independently of $q$. In fact, the functions $\kappa_{n(i, j)}$ are translates of a fixed function in $\mathscr{S}_{\alpha}^{\beta, B_{1 / 3}}$, and therefore the supremum over $q$ is smaller than a constant times the supremum over $k$ and $x$ only, if we choose $B=\max \left(B_{1 / 3}, B_{\varepsilon_{i}}\right)$ :

$$
\left\|k_{i j} \kappa_{n(i, j)}\right\|_{\rho, \delta} \leq C_{\kappa} \sup _{x, k} \frac{\left|x^{k} k_{i j} \kappa_{n(i, j)}\right|}{(A+\rho)^{k} k^{k \alpha}} .
$$

It suffices to consider this especially for $A \geq 1$ and $\alpha=1$ in which case we have

$$
\leq C_{\kappa} \sup _{x, k} \frac{\left|x^{k} k_{i j} \kappa_{n(i, j)}\right|}{k^{k}} .
$$

For $j$ large enough and by definition of $n(i, j)$ we can estimate $|x| \leq 2 j^{2}$ on the support of $\kappa_{n(i, j)}$, and with some other constant $C_{\kappa}^{\prime}$ depending only on the function $\kappa_{n(i, j)}$,

$$
\leq C_{\kappa}^{\prime} k_{i j} \sup _{k}\left(\frac{2 j^{2}}{k}\right)^{k}
$$

Continuous maximization in $k$ shows

$$
\leq C_{\kappa}^{\prime} k_{i j} \mathrm{e}^{c j^{2}}
$$

Now it is clear from their definition that $k_{i j}$ decay faster than an exponential function of any type in $j$ and thus the claim follows. 


\section{Appendix B. Basics of Indefinite InNer Product Spaces}

In this section we recall some facts about indefinite inner product, Krein and Pontryagin spaces needed in the main text. For an extensive discussion of the subject matter we refer the reader to [2, 1]. First some notations: Let $\mathcal{V}$ be a vector space equipped with an indefinite inner product $\langle.,$.$\rangle (antilinear$ in the first, linear in the second argument). The linear span of a subset $\mathcal{A}$ of vectors in $\mathcal{V}$ is denoted by $\langle\mathcal{A}\rangle$. The linear sum of subspaces $\mathcal{V}_{1}, \ldots, \mathcal{V}_{n}$ of $\mathcal{V}$ is given by $\left\langle\mathcal{V}_{1} \cup \cdots \cup \mathcal{V}_{n}\right\rangle$ and denoted by $\mathcal{V}_{1}+\cdots+\mathcal{V}_{n}$. If the spaces $\mathcal{V}_{1}, \ldots, \mathcal{V}_{n}$ are linearly independent, their linear sum is termed direct sum and denoted by $\mathcal{V}_{1}+\cdots \dot{+} \mathcal{V}_{n}$. Orthogonality with respect to $\langle.,$.$\rangle is defined, and$ denoted by the binary relation $\perp$ as usual (but clearly does not have the same strong consequences as in definite inner product spaces). If the $\mathcal{V}_{1}, \ldots, \mathcal{V}_{n}$ are mutually orthogonal, their orthogonal direct sum is denoted by $\mathcal{V}_{1}(\dot{+}) \cdots(\dot{+}) \mathcal{V}_{n}$, whereas the symbol $\oplus$ is reserved for orthogonal sums with respect to a positive definite inner product, which we will denote with $(.,$.$) , following mathematical$ convention. By positive definite we mean as usual $(x, x) \geq 0, \forall x \neq 0$, and $(x, x)=0 \Rightarrow x=0$. A subspace $\mathcal{A}$ of $\mathcal{V}$ is called positve, negative, or neutral, respectively, if one of the possibilities $\langle x, x\rangle>0,\langle x, x\rangle<0$ or $\langle x, x\rangle=0$ holds for all $x \in \mathcal{A}$, with $x \neq 0$. One sets

$$
\mathcal{V}^{++} \stackrel{\text { def }}{=}\{x \in \mathcal{V} \mid\langle x, x\rangle>0 \text { or } x=0\},
$$

and calls this subset the positive part of $\mathcal{V}$. The negative and neutral parts $\mathcal{V}^{--}$ and $\mathcal{V}^{0}$ are defined alike. A subspace $\mathcal{A}$ of $\mathcal{V}$ is called degenerate, if its isotropic part $\mathcal{A} \cap \mathcal{A}^{\perp}$ does not only consist of the zero vector. In the main text and the following we will deal merely with non-degenerate spaces, i.e., spaces with $\mathcal{V}^{\perp}=\{0\}$. A non-degenerate inner product space $\mathcal{V}$ is said to be decomposable if it admits a fundamental decomposition

$$
\mathcal{V}=\mathcal{V}^{\perp}(\dot{+}) \mathcal{V}^{+}(\dot{+}) \mathcal{V}^{-}, \quad \text { with } \mathcal{V}^{+} \subset \mathcal{V}^{++}, \mathcal{V}^{-} \subset \mathcal{V}^{--}
$$

For non-degenerate spaces the isotropic part of the decomposition vanishes. The dimension of a maximal negative definite subspace $\mathcal{V}^{-} \subset \mathcal{V}^{--}$appearing in a fundamental decomposition of a non-degenerate inner product space is called the rank of negativity of $\mathcal{V}$. As proven in [2, Corrollaries II.10.4 and IV.7.4], it is an unique positive cardinal denoted by $\varkappa^{-}(\mathcal{V})$. The rank of positivity $\varkappa^{+}(\mathcal{V})$ is defined in analogy to that. We set $\varkappa \equiv \min \left(\varkappa^{-}, \varkappa^{+}\right)$and call this number the rank of indefiniteness of $\mathcal{V}$.

Now some less trivial things about the topology of indefinite inner product spaces: A locally convex topology $\tau$ on $\mathcal{V}$ defined by a single seminorm $p$, which is then actually a norm, is called normed. If $\mathcal{V}$ is $\tau$-complete, we say that $\tau$ is a Banach topology. If $\tau$ can be defined by a quadratic norm $p(x)=(x, x)^{1 / 2}$, where $(.,$.$) is a positive definite inner product on \mathcal{V}$, then $\tau$ is called a quadratic normed topology. Again, if $\mathcal{V}$ is $\tau$-complete, then $\tau$ is termed Hilbert topology. A normed topology $\tau_{1}$ is stronger than another $\tau_{2}$, written $\tau_{1} \geq \tau_{2}$, if and only if every $\tau_{2}$-open set is also a $\tau_{1}$-open set, or equivalently the relation $p_{1}(x) \geq$ $\alpha p_{2}(x)$ holds for all $x \in \mathcal{V}$, with an $\alpha>0$. Two norms that define the same topology are called equivalent. A locally convex topology $\tau$ on $\mathcal{V}$ is called a partial majorant of the inner product if $\langle., .$.$\rangle is separately \tau$-continuous. The weak topology on $\mathcal{V}$ is the topology defined by the family of seminorms

$$
p_{y}(x) \stackrel{\text { def }}{=}|\langle y, x\rangle|, \quad \text { for all } x \in \mathcal{V} .
$$

Lemma B.1 ([2, Theorem II.2.1]). The weak topology is the weakest partial majorant on $\mathcal{V}$. If a locally convex topology on $\mathcal{V}$ is stronger than the weak topology, then it is a partial majorant. 
We will need a stronger concept of topology:

Definition B.2. A locally convex topology $\tau$ on $\mathcal{V}$ is called majorant topology, if the inner product $\langle., .$.$\rangle is jointly \tau$-continuous.

In applications, one can often restrict oneself to majorants defined by a single seminorm which majorizes the inner square, as we can see from the following result.

Lemma B.3 ([2] Lemma IV.1.1 \& 1.2]). It holds:

i) To every majorant there exists a weaker majorant defined by a single seminorm.

ii) For a locally convex topology defined by a single seminorm $p$ to be a majorant it is sufficient that $p$ dominates the inner square:

$$
|\langle x, x\rangle| \leq \alpha p(x)^{2}, \quad \alpha>0, \forall x \in \mathcal{V} .
$$

Majorant topologies, and especially majorant Hilbert topologies, have many advantages over partial majorants. Before we describe them, let us see why one would not like to use the weak topology on general indefinite inner product spaces:

Lemma B.4 ([2, Theorem IV.1.4]). The weak topology on the non-degenerate indefinite inner product space $\mathcal{V}$ is a majorant, if and only if $\operatorname{dim} \mathcal{V}<\infty$.

The indefinite inner product on a space equipped with a majorant Hilbert topology admits a simple description by the so-called metric operator.

Proposition B.5 ([2, Theorem IV.5.2]). Let $\mathcal{V}$ be an indefinite inner product space with a majorant Hilbert topology $\tau$ defined by a norm \|.\|. Then there exists a Hermitean linear operator, called metric (or Gram) operator, $J$ on $\mathcal{V}$ such that

$$
\langle x, y\rangle=(x, J y), \quad \forall x, y \in \mathcal{V},
$$

where $(.,$.$) is the positive inner product on \mathcal{V}$ that defines $\|\|$. Moreover, in this case $\mathcal{V}$ is decomposable and the fundamental decomposition can be chosen so that each of the three components is $\tau$-closed.

The spaces we want to construct in the main text should be complete in a certain sense, which we will now specify.

Definition B.6. If a non-degenerate indefinite inner product space $\mathcal{K}$ admits a decomposition

$$
\mathcal{K}=\mathcal{K}^{+}(\dot{+}) \mathcal{K}^{-}, \quad \mathcal{K}^{+} \subset \mathcal{K}^{++}, \mathcal{K}^{-} \subset \mathcal{K}^{--},
$$

such that $\mathcal{K}^{+}, \mathcal{K}^{-}$are complete with respect to the restrictions of the weak topology to them (termed intrinsically complete), then the space $\mathcal{K}$ is called a Krein space.

Krein spaces can easily be characterized:

Proposition B.7 ([2, Theorem V.1.3]). An indefinite inner product space $\mathcal{V}$ is a Krein space if and only if there exists a majorant Hilbert topology $\tau$ on $\mathcal{V}$ such that metric operator $J$ is completely invertible.

A Hilbert-space completion $\mathcal{H}$ of an indefinite inner product space $\mathcal{V}$, if it exists together with its metric operator $J$, is called the Hilbert space structure $(\mathcal{H}, J)$ associated to $\mathcal{V}$. In applications one would like to find the largest Hilbert space associated to an indefinite inner product space. For that, one considers minimal majorant topologies, i.e., topologies $\tau_{*}$ such that no majorant $\tau$ is weaker than $\tau_{*}$. Hilbert space structures given by the completion of 
$\mathcal{V}$ with respect to a minimal majorant are correspondingly called maximal. We find that the Hilbert space structure is maximal, if it leads actually to a Krein space:

Lemma B.8 ([15, Appendix A.1]). A majorant Hilbert topology leads to a maximal Hilbert space structure $(\mathcal{K}, J)$, if and only if $J$ has a bounded inverse. Given a Hilbert space structure one can always construct a maximal one.

The last statement means in effect that every space admitting some majorant Hilbert topology can be completed to a Krein space.

\section{REFERENCES}

[1] T. Ya. Azizov and I. S. Iokhvidov, Linear Operators in Spaces with an Indefinite Metric, John Wiley \& Sons, New York, Chichester, Brisbane, Toronto, 1989. 16]

[2] János Bognár, Indefinite Inner Product Spaces, Springer-Verlag, Berlin, Heidelberg, New York, 1974. 2016 16 16] 17] 17]17]17

[3] Erwin Brüning and Shigeaki Nagamachi, Hyperfunction Quantum Field Theory: Basic Structural Results, Journal of Mathematical Physics 30 (1989), no. 1, 2340-2359. 2

[4] - Closure of Field Operators, Asymptotic Abelianess, and Vacuum Structure in Hyperfunction Quantum Field Theory, Journal of Mathematical Physics 39 (1998), no. 10, 50985111, 1qp/00021800 2

[5] Daniel A. Dubin and Jan Tarski, Indefinite Metric Resulting from Regularization in the Infrared Region, Journal of Mathematical Physics 7 (1966), no. 3, 574-577. [1]

[6] I. M. Gelfand and G. E. Shilov, Generalized Functions, vol. 2, Academic Press, London, San Diego, 1964. 203 301314,14

[7] James Glimm and Arthur Jaffe, Quantum Physics: A Functional Integral Point of View, 2nd ed., Springer-Verlag, Berlin, Heidelberg, New York, 1987. [5]

[8] M. Mnatsakanova, G. Morchio, F. Strocchi, and Yu. Vernov, Irreducible Representations of the Heisenberg Algebra in Krein Spaces, Journal of Mathematical Physics 39 (1998), 2969-2982, mp_arc/95-534 35 5,5

[9] G. Morchio, D. Pierotti, and F. Strocchi, Infrared and Vacuum Structure in Two-Dimensional Local Quantum Field Theory, Journal of Mathematical Physics 31 (1990), no. 6, 1467-1477. 11] 9 [11

[10] G. Morchio and F. Strocchi, Representations of *-Algebras in Indefinite Inner Product Spaces, Stochastic Processes, Physics and Geometry: New Interplays, II (Leipzig 1999), AMS Conference Proceedings, vol. 29, American Mathematical Society, Providence, Rhode Island, 2000, pp. 491-503. 5

[11] U. Moschella and F. Strocchi, The Choice of Test Functions in Gauge Quantum Field Theory, Letters in Mathematical Physics 24 (1992), 103-113. 2

[12] C. R. Putnam, Commutation Properties of Hilbert Space Operators, Springer-Verlag, New York, 1967. 3

[13] Andreas U. Schmidt, Mathematical Problems of Gauge Quantum Field Theory: A Survey of the Schwinger Model Universitatis Iagellonicae Acta Mathematica Fasciculus XXXIV (1997), 113-134, hep-th/9707166 211]12

[14] R. F. Streater and Arthur S. Wightman, PCT, Spin and Statistics, and All That, W. A. Benjamin, London, Amsterdam, 1964. [1

[15] F. Strocchi, Selected Topics on the General Properties of Quantum Field Theory, Lecture Notes in Physics, vol. 51, World Scientific, Singapore, London, Hong Kong, 1993. 11]18

[16] Arthur S. Wightman, The Choice of Testfunctions in Quantum Field Theory, Journal of Mathematical Analysis and Applications 7B (1981), 769-791. 2

Current address: Dipartimento di Fisica E. Fermi, Università di Pisa, via Buonarroti 2, Ed. B, 56127 Pisa, Italy

Fachbereich Mathematik, Johann Wolfgang Goethe-Universität, 60054 FrankFURT AM MAIN, GERMANY, HOMEPAGE

E-mail address: aschmidtemath.uni-frankfurt.de 